Pacific Journal of Mathematic 


\title{
ARTINIAN, ALMOST ABELIAN GROUPS AND THEIR GROUPS OF AUTOMORPHISMS
}

\author{
ANNEMARIE SCHLETTE
}

The principal results of this paper are several characterizations of the class of artinian almost abelian groups. Several corollaries of the main characterization are given, one of which states that a torsion group $G$ which has a finite group of automorphisms is finite, and an abelian torsion group which has a countable automorphism group is finite. In a concluding section, the class of artinian almost abelian groups and the class of finite groups are characterized by formal group-theoretical properties.

A group is called artinian, if the minimum condition is satisfied by its subgroups. The structure of an abelian, artinian group is completely determined. Every artinian group that is known so far is an extension of an abelian, artinian group by a finite group. An extension of an abelian group by a finite group is called almost abelian. It is not known whether there exist further artinian groups. For this reason it seems to be worth while to consider the structure of artinian and almost abelian groups and we will give some characterizations of them. We will prove the following

MaIN THEOREm. The following properties of the group $G$ are equivalent:

(1) $G$ is artinian and almost abelian.

(2) (a) Every abelian subnormal subgroup of $G$ is artinian;

(b) Every infinite epimorphic image of $G$ possesses an abelian subnormal subgroup, not 1;

(c) $G$ is a torsion group.

(3) (a) Every abelian subgroup of $G$ is artinian;

(b) Every epimorphic image, not 1, of $G$ possesses an almost abelian normal subgroup, not 1.

(4) (a) $G$ is a torsion group;

(b) Every torsion group of automorphisms of $G$ is artinian and almost abelian.

(5) (a) $G$ is a torsion group;

(b) The central quotient group of $G$ is artinian and almost abelian;

(c) Primary elementary abelian groups of automorphisms of $G$ are countable.

Sections 1 to 4 deal with preliminary lemmas, some of which may 
be of independent interest. We show, for instance, that the central quotient group of $G$ is artinian and almost abelian if, and only if, $G$ is almost abelian with artinian commutator subgroup $G^{\prime}$ (Proposition 3.2). Section 5 is dedicated to the proof of the Main Theorem. A simple application of the Main Theorem yields a characterization of the artinian groups with finite central quotient group (Corollary 6.1) and of the finite groups (Corollary 6.2), both by their automorphism groups. The results of the Main Theorem and of its corollaries finally are used to characterize the class of all artinian and almost abelian groups on the one hand and the class of all finite groups on the other hand by merely formal group theoretical properties. This is done in $\S 7$ (Theorem 7.1 and Theorem 7.2).

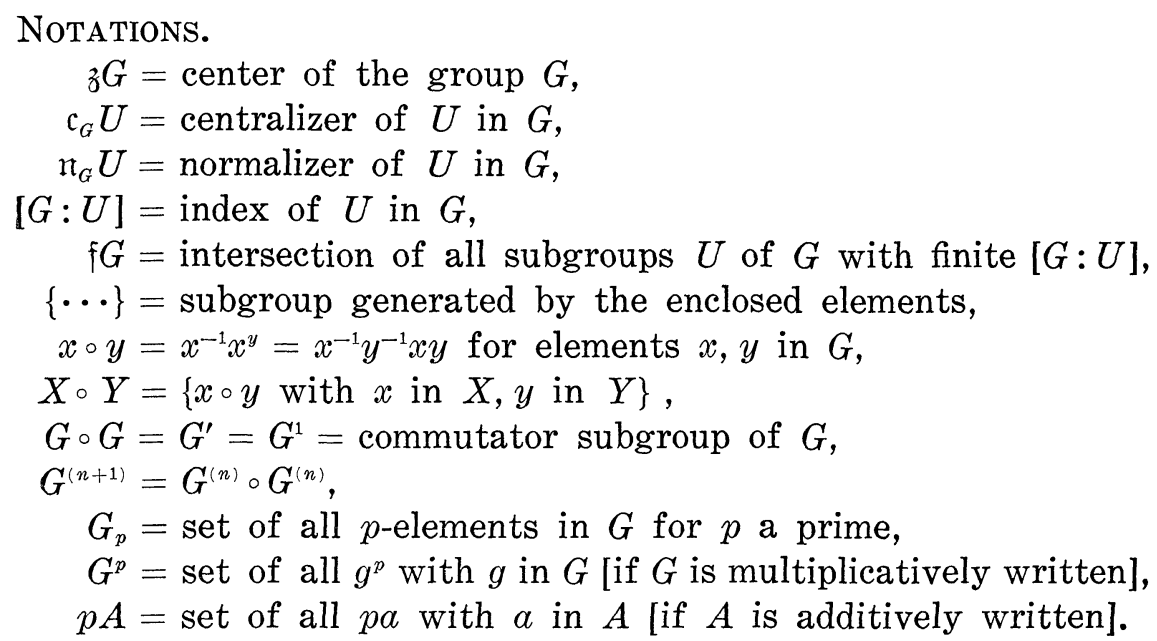

Definitions. A factor of $G$ is an epimorphic image of a subgroup of $G$.

The socle of an abelian $p$-group is the set of its elements of order $p$ or 1 .

The group $G$ is called

artinian, if its subgroups satisfy the minimum condition;

almost abelian, if it possesses an abelian [characteristic] subgroup of finite index;

soluble, if $G^{(n)}=1$ for almost all $n$;

locally finite, if every finitely generated subgroup of $G$ is finite; hyper (almost) abelian, if every epimorphic image, not 1, of $G$ possesses an (almost) abelian normal subgroup, not 1 .

$$
\left.\begin{array}{c}
\mathfrak{h} a G \\
\mathfrak{a} a G \\
\mathfrak{I} G G
\end{array}\right\}=\text { product of all }\left\{\begin{array}{l}
\text { hyperabelian } \\
\text { almost abelian } \\
\text { locally finite }
\end{array}\right\} \text { normal subgroups of } G \text {. }
$$


An abelian group $A$ is called divisible (radicable), if $p A=A\left(A^{p}=A\right)$ for all primes $p$, and reduced, if it possesses no divisible (radicable) subgroup $\neq 0(\neq 1)$.

1. Lemmas on abelian groups. In this chapter we consider (additively written) abelian groups $A$. Any homomorphism $\sigma$ of $A$ maps the element $x$ in $A$ upon $x \sigma$.

Lemma 1.1. A reduced abelian p-group $A$ with finite $A / p A$ is finite.

Proof. Since $A / p A$ is finite, there exists a finitely generated subgroup $F$ of $A$ with $A=F+p A$. Since finitely generated, abelian $p$-groups are finite, $F$ is finite. Therefore there exists a positive integer $n$ with $p^{n} F=0$. Hence

$$
p^{n} A=p^{n} F+p^{n+1} A=p\left(p^{n} A\right)
$$

is divisible. But $A$ is reduced, so that $p^{n} A=0$. From $A=F+p A$ we deduce

$$
\begin{aligned}
A & =F+\left[p F+p^{2} A\right]=F+p^{2} A=\cdots= \\
& =F+p^{n} A=F,
\end{aligned}
$$

so that $A$ is finite.

Lemma 1.2. If $A$ is an abelian, reduced, infinite p-group and if $B$ is an abelian $p$-group $\neq 0$, then $\operatorname{Hom}(A, B)$ contains an uncountably infinite, elementary abelian p-subgroup.

Proof. Since $A$ is an abelian, reduced, infinite $p$-group, $A / p A$ is infinite, by Lemma 1.1. Since $B \neq 0$, it contains a subgroup $Z$ of order $p$. Now

$$
\operatorname{Hom}(A, Z) \subseteq \operatorname{Hom}(A, B)
$$

and

$$
\operatorname{Hom}(A, Z) \cong \operatorname{Hom}(A / p A, Z),
$$

which is an uncountably infinite, elementary abelian p-group, (cf. [5], p. 206; E, and p. 208; Lemma 54.2).

Lemma 1.3. (a) If $A$ and $B$ are abelian, artinian groups, then the torsion subgroup of $\operatorname{Hom}(A, B)$ is finite.

(b) Torsion groups of automorphisms of an abelian, artinian group are finite. 
Proof. The abelian artinian group $A$ is a direct sum

$$
A=F \oplus D
$$

of a finite group $F$ and a divisible group $D$, (cf. [5], p. 65; Th. 19.2). Hence there exists a positive integer $n$ with $n F=0$. Let $X$ be the subgroup of all $x$ in $B$ with $n x=0$. Since $B$ is abelian and artinian, it is a consequence of ([5], p. 65; Th. 19.2) that $X$ is finite.

If $\sigma$ is a torsion element of order $i$ in $\operatorname{Hom}(A, B)$, then

$$
D \sigma=(i D) \sigma=D(i \sigma)=D 0=0
$$

and

$$
n(A \sigma)=(n F \oplus n D) \sigma=n(D \sigma)=0,
$$

so that $A \sigma \subseteq X$. Therefore the torsion subgroup of $\operatorname{Hom}(A, B)$ is essentially the same as a group of homomorphisms of the finite group $A / D \cong F$ into the finite group $X$, which implies its finiteness. This proves (a), and (b) is a consequence of (a) if we let $A=B$.

LEMma 1.4. If the abelian group $A$ is the direct sum of infinitely many subgroups $A_{\nu} \neq 0$, then $A$ possesses an uncountably infinite, elementary abelian 2-group of automorphisms.

The simple proof of this lemma may be left to the reader.

\section{Stabilizing automorphism groups.}

Proposition 2.1. Suppose $B$ is a normal subgroup of the group $G$ and $A$ is a subgroup of $G$ with $A \subseteq B \cap z G$. Let $\Sigma=\Sigma_{G}(A, B)$ be the group of all automorphisms $\sigma$ of $G$ which fix every element in $G / A$ and in $B$. Then

$$
\Sigma_{G}(A, B) \cong \operatorname{Hom}(G / B, A) \cong \operatorname{Hom}\left(G / B G^{\prime}, A\right) .
$$

Proof. If $\sigma$ is an automorphism in $\Sigma$, then $\sigma-1$ is a single valued mapping of $G$ into $A$. If $x$ and $y$ are elements in $G$, then

$$
(x y)^{\sigma-1}=x^{\sigma} y^{\sigma} y^{-1} x^{-1}=x^{\sigma} y^{\sigma-1} x^{-1}=x^{\sigma-1} y^{\sigma-1},
$$

since $y^{\sigma-1}$ belongs to $A \subseteq z G$. Therefore $\sigma-1$ is a homomorphism of $G$ into $A$, whose kernel contains $B$. Thus

(a) If $\sigma$ is an element in $\Sigma$, then $\sigma-1$ induces a homomorphism $\sigma^{*}$ of $G / B$ into $A$ with

$$
(B g)^{\sigma^{*}}=g^{\sigma-1} \text {. }
$$


Let $\alpha, \beta$ be two automorphisms in $\Sigma$ and $g$ be any element in $G$. Then

$$
g^{\alpha^{\beta-1}}=g^{\alpha^{\beta}}\left(g^{-1}\right)^{\beta} g^{\beta} g^{-1}=\left(g^{\alpha-1}\right)^{\beta} g^{\beta-1}=g^{\alpha-1} g^{\beta-1},
$$

since $g^{\alpha-1}$ belongs to $A \subseteq B$ and every element in $B$ is a fixed element of $\beta$. Therefore

$$
(\alpha \beta)^{*}=\alpha^{*}+\beta^{*} \quad \text { for } \alpha, \beta \text { in } \Sigma,
$$

so that $\sigma \rightarrow \sigma^{*}$ is a homomorphism of $\Sigma$ into $\operatorname{Hom}(G / B, A)$. If $\sigma^{*}=0$ and $g$ is an element in $G$, then

$$
1=(B g)^{\sigma^{*}}=g^{\sigma-1} \text {. }
$$

This implies $g=g^{\sigma}$ for every $g$ in $G$, so that $\sigma=1$. Hence

(b) The mapping $\sigma \rightarrow \sigma^{*}$ is a monomorphism of $\Sigma$ into Hom $(G / B, A)$.

For every $\gamma$ in $\operatorname{Hom}(G / B, A)$ define the mapping $\delta$ of $G$ into $G$ by the rule

$$
g^{\bar{o}}=(B g)^{\gamma} g \quad \text { for every } g \text { in } G .
$$

This is clearly a single valued mapping of $G$ into itself.

If $x, y$ are two elements in $G$, then

$$
(x y)^{\delta}=(B x y)^{\gamma} x y=(B x)^{\gamma}(B y)^{\gamma} x y=(B x)^{\succ} x(B y)^{\gamma} y=x^{\delta} y^{\delta}
$$

since $(B y)^{r}$ belongs to $A \subseteq \jmath G$. Thus $\delta$ is an endomorphism of $G$. If $x^{\tilde{o}}=1$, then $(B x)^{\gamma} x=1$, so that $x$ belongs to $B$ (since $(B x)^{r}$ is in $A \subseteq B$ ). This implies $(B x)^{\gamma}=B^{\gamma}=1$ so that $x=1$. Hence $\delta$ is a monomorphism of $G$ into $G$. Because of $B^{r}=1$ and $(B g)^{r} \in A$ it is clear that $\delta$ induces the identity in both $B$ and $G / A$. Now let $g$ be any element in $G$ and let

$$
x=\left(B g^{-1}\right) r g .
$$

Then

$$
x^{\tilde{o}}=(B x)^{\gamma} x=\left[B\left(B g^{-1}\right)^{\gamma} g\right]^{\gamma}\left(B g^{-1}\right)^{\gamma} g=(B g)^{\gamma}\left[(B g)^{\gamma}\right]^{-1} g=g
$$

since $\left(B g^{-1}\right)^{r}$ belongs to $A \subseteq B$. This shows that $\delta$ is an automorphism of $G$ which belongs to $\Sigma$. Finally

$$
g^{\grave{o}-1}=(B g)^{\gamma} \quad \text { for every } g \text { in } G,
$$

shows $\delta^{*}=\gamma$ and

(c) $\sigma \rightarrow \sigma^{*}$ is an isomorphism of $\Sigma$ onto $\operatorname{Hom}(G / B, A)$.

The isomorphy of $\operatorname{Hom}(G / B, A)$ and $\operatorname{Hom}\left(G / B G^{\prime}, A\right)$ is an immediate consequence of the commutativity of $A$. 
LEMMA 2.2. If the group $G$ possesses a characteristic subgroup $A$ such that the torsion groups of automorphisms of $A$ and of $G / A$ are finite, then every torsion group $\Gamma$ of automorphisms of $G$ is almost abelian: The subgroup $\Delta$ of $\Gamma$ which fixes every element in $A$ and in $G / A$ is abelian and has finite index in $\Gamma$.

Proof. Let $\Gamma$ be a torsion group of automorphisms of $G$. Denote by $\Gamma_{A}$ resp. $\Gamma_{G / A}$ the set of all those automorphisms in $\Gamma$, which induce the identity in $A$ and in $G / A$ respectively. Since $A$ is characteristic in $G$, the sets $\Gamma_{A}$ and $\Gamma_{G / A}$ are both normal subgroups of $\Gamma$. Now $\Gamma / \Gamma_{A}$ resp. $\Gamma / \Gamma_{G / A}$ is essentially the same as the group of automorphisms of $A$ resp. $G / A$, induced by $\Gamma$. Therefore it is (essentially) a torsion group of automorphisms of $A$ resp. $G / A$, and hence, by hypothesis, finite. If $\Delta=\Gamma_{A} \cap \Gamma_{G / A}$, then $\Delta$ is a normal subgroup of $\Gamma$ with finite $\Gamma / \Delta$. By its definition, $\Delta$ is a subgroup of the stabilizer of the characteristic subgroup $A$ of $G$. As such it is known to be abelian, see ([7], p. 88; Satz 19). This proves Lemma 2.2.

3. Central quotient group and commutator subgroup. In this chapter we aim at characterizing the groups with almost abelian, artinian central quotient group (Proposition 3.2).

LEMMA 3.1. If $G$ is a group with abelian, radicable, not torsionfree central quotient group $G / z G$, then $G$ is abelian.

Proof. Suppose that $G$ is not abelian. Then $G / z G \neq 1$ and since it is not torsionfree there exists a subgroup $W / z G$ in $G / z G$ of order a prime $p$. Now $G^{\prime} \subseteq z G \subset W$, so that $W$ is normal in $G$. Since $G / z G$ is abelian, it follows that $W \circ G \leqq z G$ and that

$$
w(w \circ g)=(w \circ g) w \text { for every } w \in W, g \in G .
$$

Since $w^{p}$ also belongs to $z G$, it is centralized by every element in $G$. Therefore it follows from $w^{g}=w(w \circ g)$ that

$$
w^{p}=\left(w^{p}\right)^{g}=\left(w^{g}\right)^{p}=(w(w \circ g))^{p}=w^{p}(w \circ g)^{p} .
$$

Hence

$$
(w \circ g)^{p}=1 \quad \text { for every } \quad w \in W, g \in G .
$$

By complete induction we get

$$
w^{g^{i}}=w(w \circ g)^{i} \text { for every positive integer } i
$$

and in particular

$$
w^{g^{p}}=w(w \circ g)^{p}=w,
$$


which shows

$$
G^{\prime} G^{p} \cong G^{p}{ }^{p} G \leqq \mathrm{c}_{G} W .
$$

Therefore the epimorphic image $G / c_{G} W$ of $G / z G$ is an elementary abelian $p$-group. Since elementary abelian $p$-groups $\neq 1$ possess finite nontrivial epimorphic images, $G / c_{G} W$ is equal to 1 , because $G / z G$ is radicable. Hence $\mathfrak{c}_{G} W=G$, implying $W \subseteq z G \subset W$, a contradiction. Therefore $G$ is abelian.

Definition. $\mathrm{f} G=$ intersection of all subgroups $U$ of $G$ with finite index $[G: U]$. This is a well determined characteristic subgroup of $G$.

Proposition 3.2. The following properties of the group $G$ are equivalent:

(i) $G / 3 G$ is artinian and almost abelian.

(ii) If $F$ is the uniquely determined characteristic subgroup of $G$ with $z G \subseteq F$ and $F / z G=\mathfrak{f}(G / z G)$, then $F$ is abelian, $G / F$ is finite, and $G \circ F$ is artinian.

(iii) There exists an abelian normal subgroup $A$ of $G$ with finite $G / A$ and artinian $G \circ A$.

(iv) $G$ is almost abelian and $G^{\prime}$ is artinian.

Proof. (i) $\rightarrow$ (ii). Since $G / z G$ is artinian and almost abelian, application of ([1], pp. $3,4,22(\mathrm{~B}))$ on $G / z G$ shows

(a) $G / F=(G / z G) / \mathfrak{f}(G / z G)$ is finite and $\mathrm{f}(G / z G)=F / z G$ does not possess finite epimorphic images. $F / z G$ is abelian because $G / z G$ is almost abelian. Hence $F^{\prime} \subseteq z G \subseteq z F \subseteq F$ and therefore

(b) $F / z F$ is an abelian radicable torsion group. Apply Lemma 3.1 on $F$ to see

(c) $F$ is abelian.

Let $\Gamma$ be the group of automorphisms which is induced in $F$ by G. Then $\Gamma \cong G / c_{G} F$ is finite because $F \cong c_{G} F$ by (c), and $G / F$ is finite, by (a). Every automorphism $\gamma$ in $\Gamma$ fixes every element in $z G$. Hence $\gamma-1$ is an endomorphism of the abelian group $F$ whose kernel contains $z G$. Consequently $F^{i-1}$ is an epimorphic image of the artinian group $F / z G$ and as such $F^{\gamma-1}$ is artinian. Since $\Gamma$ is finite and $F$ is abelian,

$$
F^{\Gamma-1}=\prod_{\gamma \in \Gamma} F^{\gamma-1}
$$

is likewise artinian. From the definition of $\Gamma$,

$$
G \circ F=F^{r-1}
$$

so that 
(d) $G \circ F$ is artinian.

(ii) $\rightarrow$ (iii). Let $A=F$.

(iii) $\rightarrow$ (iv). It is an immediate consequence of (iii) that $G$ is almost abelian. Since $A$ is normal in $G$, so is $G \circ A$. Denote by $\sigma$ the canonical epimorphism of $G$ upon $H=G /(G \circ A)$. Then $B=A^{\sigma}$ is an abelian normal subgroup of $H$ with finite $H / B$, as $G / A$ is finite. From $H \circ B=(G \circ A)^{\sigma}=1$ we get $B \subseteq{ }_{z} H$, so that $H /{ }^{z} H$ is finite. This implies the finiteness of $H^{\prime}$, see $\left([6]\right.$, p. 443; 15.1.13). But $H^{\prime}=G^{\prime \sigma} \cong$ $G^{\prime} /(G \circ A)$, so that $G^{\prime}$ is artinian as an extension of the artinian group $G \circ A$ by the finite group $H^{\prime}$.

(iv) $\rightarrow$ (iii). Obvious.

(iii) $\rightarrow$ (i ). The group $\Gamma$ of automorphisms of $A$ which are induced in $A$ by $G$ is isomorphic to $G / c_{G} A$ and it is finite since $A \subseteq c_{G} A$ and $G / A$ is finite. If $\gamma$ is an automorphism in $\Gamma$, then $\gamma-1$ is an endomorphism of the abelian group $A$ with $A^{\gamma-1} \subseteq G \circ A$. It follows that $A^{\gamma-1}$ is artinian and $A^{\gamma-1} \cong A / K(\gamma)$, where $K(\gamma)$ denotes the kernel of $\gamma$, i.e., $K(\gamma)=\mathfrak{c}_{A} g$ if $g$ induces $\gamma$. Now

$$
A \cap \jmath G=\bigcap_{\gamma \in \Gamma} K(\gamma) .
$$

Therefore $A /(A \cap z G)$ is isomorphic to a subgroup of the direct product of the finitely many artinian groups $A / K(\gamma)$ and as such $A /(A \cap z G) \cong$ $A_{z} G / \xi G$ is itself artinian. $G / A_{z} G$ is finite as an epimorphic image of $G / A$. Finally $G / z G$ is an extension of the abelian artinian group $A_{z} G / z G$ by the finite group $G / A_{\mathfrak{z}} G$. Hence $G / \bar{z} G$ is artinian and almost abelian.

4. Products of normal subgroups. In this section we shall establish a few lemmas on products of normal subgroups, which are needed later. In this context see also Corollary 6.4. of $G$.

Definition. $\mathfrak{h a} G=$ product of all hyperabelian normal subgroups

This is a well determined characteristic subgroup of $G$.

LEMMA 4.1. If every abelian subnormal subgroup of the torsion group $G$ is artinian, then

(a) haG is artinian and soluble;

(b) haG contains every hyperabelian subnormal subgroup of $G$;

(c) 1 is the only hyperabelian subnormal subgroup of $G / \mathfrak{b a} G$;

(d) $\quad \mathfrak{h a}(G / \mathfrak{h a} G)=1$.

Proof. If $S$ is a subnormal subgroup of $G$, then every abelian subnormal subgroup of $\mathfrak{h a} S$ is a subnormal subgroup of $G$ and hence, by hypothesis, artinian. Nontrivial epimorphic images of haS possess 
by definition hyperabelian normal subgroups, not 1 , and consequently abelian subgroups, not 1 ; and $\mathfrak{h a} S$ is a torsion group, as is $G$. Apply ([4], p. 345, Satz 6.1, equivalence of (1) and (2)) to haS to show that (e) ha $S$ is artinian and soluble for every subnormal subgroup $S$ of $G$. This implies (a) in the particular case $S=G$.

Since $S$ is subnormal in $G$, there exists a finite chain of subgroups $S_{i}$ of $G$ with

$$
S=S_{0}, S_{i} \text { normal in } S_{i+1}, S_{n}=G .
$$

If $S$ is hyperabelian, then $S=\mathfrak{h a} S_{0} . \quad B y(e), \mathfrak{h a} S_{i}$ is a soluble, hence hyperabelian characteristic subgroup of the normal subgroup $S_{i}$ of $S_{i+1}$. Hence $\mathfrak{b a} S_{i} \subseteq \mathfrak{h a} S_{i+1}$, showing

$$
S=\mathfrak{h a} S_{0} \subseteq \cdots \subseteq \mathfrak{h} \mathfrak{a} S_{n}=\mathfrak{h} \mathfrak{a} G,
$$

so that $\mathfrak{h a} G$ contains every hyperabelian subnormal subgroup of $G$, proving (b). Now let $T=S / \mathfrak{h a G}$ be a hyperabelian subnormal subgroup of $G / \mathfrak{h a} G$. Then $S$ is as an extension of the, by (a), soluble group ha $G$ by the hyperabelian group $T$ hyperabelian. Since $S$ is subnormal in $G$, it follows from (b) that $S \subseteq \mathfrak{h a} G$. Hence $T=1$, proving (c).

$(d)$ is an immediate consequence of (c).

Lemma 4.2. If $N$ is a product of minimal normal subgroups of the group $G$, then $N$ is a direct product of minimal normal subgroups of $G$.

This is proven by the customary lattice theoretical arguments; see for instance ([8], p. 208; Basis Theorem of Lattice Theory).

LEMmA 4.3. If a minimal normal subgroup $M$ of $G$ is contained in a product $N$ of finite normal subgroups of $G$, then $M$ is finite.

Proof. Let $t \neq 1$ be an element in $M$. Since $t$ belongs to the product $N$ of finite normal subgroups of $G$, there exists a finite number $n$ of finite normal subgroups $F_{i}$ of $G$ with $F_{i} \leqq N$ and

$$
t \in \prod_{i=1}^{n} F_{i}=F .
$$

Clearly $F$ is a finite normal subgroup of $G$ with $t$ in $F \subseteq N$. Hence $1 \neq M \cap F$ and because of the minimality of $M$ we have $M=M \cap F \subseteq F$, so that $M$ is finite.

LEMma 4.4. If 1 is the only abelian normal subgroup of $G$, then the following properties of the product $N$ of finite normal subgroups 
of $G$ are equivalent:

(i) $N$ is finite.

(ii) $N$ contains but a finite number of minimal normal subgroups of $G$.

(iii) The product $P$ of all the minimal normal subgroups of $G$, contained in $N$, is the direct product of finitely many minimal normal subgroups of $G$.

Proof. Evidently, (ii) is a consequence of (i) and it follows from Lemma 4.2 that $P$ is a direct product of minimal normal subgroups of $G$, therefore (iii) is a consequence of (ii).

Assume that (iii) holds. Then application of Lemma 4.3 shows that

$$
\text { the normal subgroup } P \text { of } G \text { is finite. }
$$

Since $P$ is finite, the group of automorphisms, induced in $P$ by $G$, which is essentially the same as $G / c_{G} P$, is finite, too. Since $z P=$ $P \cap \mathfrak{c}_{G} P$ is an abelian normal subgroup of $G$ and since, by hypothesis, 1 is the only abelian normal subgroup of $G$, we get

$$
P \cap \mathfrak{c}_{G} P=1 \text { and } G / \mathfrak{c}_{G} P \text { is finite. }
$$

If $N \cap \mathfrak{c}_{G} P \neq 1$, then there would exist a finite number of finite normal subgroups $E_{1}, \cdots, E_{n}$ of $G$ with $E_{i} \subseteq N$ and

$$
W=\mathfrak{c}_{G} P \cap \prod_{i=1}^{n} E_{i} \neq 1 \text {. }
$$

It follows that $W$ is a finite normal subgroup, not 1 , of $G$. Hence $W$ contains a minimal normal subgroup $V$ of $G$. Since

$$
V \cong W \cong \prod_{i=1}^{n} E_{i} \subseteq N
$$

this minimal normal subgroup $V$ is part of $P$. Hence

$$
1 \subset V \subseteq P \cap W \leqq P \cap c_{G} P=1,
$$

by $\left({ }^{\prime \prime}\right)$ which is a contradiction. Therefore

$$
N \cap \mathfrak{c}_{G} P=1
$$

which implies that

$$
N=N /\left(N \cap \mathfrak{c}_{G} P\right) \cong N c_{G} P / \mathfrak{c}_{G} P \subseteq G / c_{G} P
$$

is finite, by (").

Thus (i) is a consequence of (iii), proving Lemma 4.4.

5. Proof of the Main Theorem. The proof of the equivalence 
of (1), (2), and (3) was given in a course of lectures held by R. Baer at New Mexico State University in the fall term 1967.

Proof of the equivalence of (1) and (2). Evidently (1) implies (2.a) and (2.c). Furthermore every epimorphic image of an almost abelian group is almost abelian, which implies (2.b).

Assume conversely the validity of conditions (2.a-c). Then Lemma 4.1 shows and

(d) $\mathfrak{h a G}$ is artinian and soluble,

(e) 1 is the only hyperabelian subnormal subgroup of $G / \mathfrak{h} a G$. Combine (e) and (2.b) to get

(f) $G / \mathfrak{h a} G$ is finite.

Therefore, by (d) and (f),

( $\mathrm{g}) \quad G$ is artinian.

Since haG is almost abelian by (d), cf. ([4], p. 345, Satz 6.1 and its proof), there exists an abelian characteristic subgroup $C$ of $\mathfrak{h a} G$ with finite $\mathfrak{h a} G / C$. Then $C$ is an abelian characteristic subgroup of $G$ and $G / C$ is finite, by (f). Thus

( h ) $G$ is almost abelian

and we have proven the equivalence of (1) and (2).

Proof of the equivalence of (1) and (3). If $G$ is artinian and almost abelian then every subgroup of $G$ is artinian and every epimorphic image of $G$ is almost abelian, proving that (3) is a consequence of (1).

Assume the validity of (3.a) and (3.b). Then, by (3.a), every cyclic subgroup of $G$ is finite. Hence

(c) $\mathrm{G}$ is a torsion group.

Lemma 4.1 shows and

(d) $\mathfrak{h a} G$ is artinian and soluble,

(e) 1 is the only abelian subnormal subgroup of $H=G / \mathfrak{h a} G$.

If $A$ is an abelian subgroup of $H$ then there exists a subgroup $B$ of $G$ with haG $\subseteq B$ and $A=B / \mathfrak{h a} G$. By (d), $B$ is soluble and hence hyperabelian and by (3.a) every abelian subgroup of $B$ is artinian. Hence, by Lemma 1.3. (b), every torsion group of automorphisms of every abelian subgroup of $B$ is finite. Because of (c) we may apply ([4], p. 345, Satz 6.1, equivalence of (1) and (4)) on $B$. This shows that $B$ is artinian. Hence $A$ is artinian, too, proving

(f) Abelian subgroups of $H$ are artinian.

Let $F$ be the product of all finite normal subgroups of $H$ and let $P$ be the product of all minimal normal subgroups of $H$ which are contained in $F$. Application of Lemma 4.2 shows that $P$ is a direct 
product of minimal normal subgroups $X$ of $H$. Since $H$ is a torsion group by (c), we may pick in every $X$ an element $e(X)$ of order a prime. Then the subgroup $S$ generated by these $e(X)$ is a direct product of the cyclic groups $\{e(X)\}$, each of order a prime. Thus $S$ is abelian and hence, by (f), artinian. Therefore and since the elements of $S$ are of squarefree order, $S$ is finite, cf. ([5], p. 68; Exercise 19). Hence

(g) $P$ is a direct product of finitely many minimal normal subgroups of $H$. Because of (e) and (g) we may apply Lemma 4.4 to show that

(h) $F$ is finite.

Since $H / \mathfrak{c}_{H} F$ is essentially the same as the group of automorphisms induced by $H$ in $F$, it is finite, as $F$ is and, by (e), $1=z F=F \cap c_{H} F$. Thus

(i) $H / c_{H} F$ is finite and $1=F \cap c_{H} F$.

Assume by way of contradiction that $H$ is infinite. Then $F \neq H$ because of (h), so that $H / F$ is an epimorphic image, not 1 , of $G$. By (3.b) there exists an almost abelian normal subgroup, not 1, of $H / F$. Consequently there exists a normal subgroup $V$ of $H$ with

$$
F \subset V \text { and almost abelian } V / F \text {. }
$$

Apply (i) to see that

$$
V / \mathfrak{c}_{V} F=V /\left(V \cap \mathfrak{c}_{H} F\right) \cong V \mathfrak{c}_{H} F / \mathfrak{c}_{H} F \cong H / \mathfrak{c}_{H} F
$$

is finite and

$$
\mathfrak{c}_{V} F=\left(V \cap \mathfrak{c}_{H} F\right) /\left(F \cap \mathfrak{c}_{H} F\right) \cong F\left(V \cap \mathfrak{c}_{H} F\right) / F \subseteq V / F
$$

is almost abelian.

Because of (") $\mathfrak{c}_{V} F$ possesses an abelian characteristic subgroup $C$ with finite $c_{V} F / C$. As a characteristic subgroup of the normal subgroup $\mathfrak{c}_{V} F$ of $H$ the group $C$ is normal in $H$. Hence $C=1$ by (e), which shows that $\mathfrak{c}_{V} F$ is finite. Hence $V$ is a finite normal subgroup of $H$, by ('), whence $V \subseteq F \subset V$, a contradiction, proving

(k) $H=G / \mathfrak{h} \mathfrak{a} G$ is finite.

Combination of $(\mathrm{d})$ and $(\mathrm{k})$ shows that

(1) $G$ is artinian and almost abelian.

This completes the proof of the equivalence of (1) and (3) of the Main Theorem.

Proof of the equivalence of (1), (4), and (5).

$(1) \rightarrow(4)$. Every artinian group is a torsion group, hence (4.a) is valid. If $G$ is furthermore almost abelian, then there exists an abelian characteristic subgroup $A$ of $G$ with finite $G / A$. Since $A$ is artinian 
as a subgroup of the artinian group $G$, its torsion groups of automorphisms are finite, by Lemma 1.3. Therefore Lemma 2.2 is applicable, showing that every torsion group $\Gamma$ of automorphisms of $G$ is almost abelian. Define $\Delta$ as in Lemma 2.2.

The set $S$ of all the elements of squarefree order in the artinian abelian group $A$ is a finite characteristic subgroup of $A$; cf. ([5], p. 68; Exercise 19). Let $\Sigma$ be the set of all elements of squarefree order in $\Delta$. If $\sigma$ is an element of order $n$ in $\Sigma$ and if $x$ is an element in $G$, then $x^{\sigma-1}$ belongs to $A$ and is therefore a fixed element of $\sigma$. Thus $x^{\sigma}=\left(x^{\sigma-1}\right) x$ and, by complete induction,

$$
x^{\sigma^{i}}=\left(x^{\sigma-1}\right)^{i} x \quad \text { for every integer } i \geqq 0,
$$

so that in particular

$$
x=x^{\sigma^{n}}=\left(x^{\sigma-1}\right)^{n} x .
$$

This implies

$$
\left(x^{\sigma-1}\right)^{n}=1 \text { for every } x \text { in } G .
$$

Hence $x^{\sigma-1}$ is an element of squarefree order in $A$ and belongs therefore to $S$. Thus

$$
G^{\sigma-1} \cong S \text { for all } \sigma \text { in } \Sigma \text {. }
$$

Every $\sigma-1$, with $\sigma$ in $\Sigma$, induces a single valued mapping of $G / A$ into $S$, as

$$
(a x)^{\sigma-1}=a x^{\sigma-1} a^{-1}=x^{\sigma-1} \text { for } a \text { in } A, x \text { in } G,
$$

since $a^{\sigma}=a$ and $x^{\sigma-1}$ both belong to the abelian group $A$. If $\sigma^{\prime}$ and $\sigma^{\prime \prime}$ are two elements in $\Sigma$, then $\sigma^{\prime}-1$ and $\sigma^{\prime \prime}-1$ induce the same mapping of $G / A$ into $S$ if, and only if, $\sigma^{\prime}=\sigma^{\prime \prime}$. Since $G / A$ and $S$ are finite there exists only a finite number of single valued mappings of $G / A$ into $\mathrm{S}$. This implies that $\Sigma$ is finite. As $\Delta$ is an abelian torsion group, application of $([5]$, p. 68; Exercise 19) shows that $\Delta$ is artinian.

Since $\Gamma / \Delta$ is finite, $\Gamma$ is artinian and almost abelian. Hence (4) is a consequence of (1).

$(4) \rightarrow(5)$. Since $G$ is a torsion group, so is $G / z G$; and $G / z G$ is essentially the same as the group of inner automorphisms of $G$, hence it is artinian and almost abelian, by (4.b). So (5) is but a weak form of (4).

$(5) \rightarrow(1)$. Because of (5.b) Proposition 3.2 is applicable, which shows

(d) $G^{\prime}$ is artinian

and 
(e) There exists an abelian characteristic subgroup $F$ of $G$ with $F \supseteqq z G$ and finite $G / F$. Thus $G$ is almost abelian.

By (5.a), $F$ is a torsion group, so that it is locally finite, since it is abelian. Hence $G$ is an extension of the locally finite group $F$ by the finite group $G / F$, showing

(f ) $G$ is locally finite.

Since $G / F$ is finite there exists a finitely generated subgroup $E$ of $G$ with $G=F E$. Because of (f), $E$ is finite:

(g) There exists a finite subgroup $E$ of $G$ with $G=F E$.

Consider an abelian direct factor $A$ of $G$. Every group of automorphisms of $A$ and of every direct factor of $A$ is isomorphic to a group of automorphisms of $G$. If $A$ would have infinitely many primary components, it would possess an uncountably infinite, elementary abelian 2-group of automorphisms (Lemma 1.4), contradicting (5.c). For each primary component $A_{p}$ of $A$ we have, by ([5], p. 63; Th. 18.2),

$$
A_{p}=B \otimes C,
$$

where $B$ is radicable and $C$ is reduced. As a radicable torsion group $B$ is the direct product of groups of type $p^{\infty}$. If there were infinitely many, $B$ would admit an uncountably infinite, elementary abelian 2-group of automorphisms (Lemma 1.4) which again is impossible because of (5.c). Hence, by ([5], p. 65; Th. 19.2), $B$ is artinian. If $C \neq 1$, then there exists a subgroup $Z$ of order $p$ in $C$. By Proposition 2.1, Hom $(C / Z, Z)$ is isomorphic to a group of automorphisms of $C$. Now $C / Z$ is reduced, as $C$ is. If $C$ were infinite, $C / Z$ would be infinite and, by Lemma 1.2 , Hom $(C / Z, Z)$ would contain an uncountably infinite, elementary abelian $p$-subgroup, which would be isomorphic to a group of automorphisms of $C$. This contradiction shows that $C$ is finite. Hence, by ([5], p. 65; Th. 19.2), we have

(h) Every abelian direct factor of $G$ is artinian.

We derive from (d), (e), and (g) that $G^{\prime} E$ is artinian and almost abelian. Therefore the set $\omega$ of primes which are orders of elements in $G^{\prime} E$ is finite, see for instance ([3]; p. 13, Bemerkung 2.2, (A)).

Denote by $F_{\omega}$ the set of all $\omega$-elements in $F$ and by $F_{\omega^{\prime}}$ the set of all elements in $F$ of order prime to every prime in $\omega$. Since $F$ is an abelian torsion group,

$$
F=F_{\omega} \otimes F_{\omega^{\prime}} \cdot
$$

Both, $F_{\omega}$ and $F_{\omega^{\prime}}$ are characteristic subgroups of the characteristic subgroup $F$ of $G$, hence they are characteristic in $G$. By its definition, $F_{\omega} G^{\prime} E$ is a normal $\omega$-subgroup of $G$. Furthermore

$$
F_{\omega^{\prime}} \cap F_{\omega} G^{\prime} E=1,
$$


since the elements in these two groups have relatively prime order. Thus, by (g),

$$
G=F E=F_{\omega^{\prime}} F_{\omega} E=F_{\omega^{\prime}} \otimes F_{\omega} G^{\prime} E .
$$

Application of $(\mathrm{h})$ shows that $F_{\omega^{\prime}}$ is artinian, implying the finiteness of $\omega^{\prime}$. Therefore

(i) There exist only finitely many primes which are orders of elements in $G$.

The product $R$ of all the radicable subgroups of $z G$ is a radicable characteristic subgroup of $z G$ and hence of $F$. As $R$ is a torsion group it is a product of groups of type $Z\left(p^{\infty}\right)$ for various primes $p$. Let $R_{1}$ be maximal among the direct products of those $Z\left(p^{\infty}\right)$ in $R$, which have a nontrivial intersection with $G^{\prime} E$. Then clearly the elements of squarefree order of $R_{1}$ lie in $G^{\prime} E$. This implies that $R_{1}$ is artinian. Moreover, $R_{1}$ is radicable as it is the direct product of radicable groups. Therefore $R_{1}$ is a direct factor of $R$, cf. ([5], p. 62; Th. 18.1),

$$
R=R_{1} \otimes R_{2}
$$

where $R_{2}$, as an epimorphic image of $R$ is likewise radicable. Let $x$ be an element of prime order $p$ in $R_{2} \cap G^{\prime} E$. Then $x$ is contained in a subgroup $V \cong R_{2}$ of type $Z\left(p^{\infty}\right)$ and $x \neq 1$ would imply $V \cap G^{\prime} E \neq 1$, a contradiction to the maximality of $R_{1}$. Therefore

$$
R_{2} \cap G^{\prime} E=R_{2} \cap\left(F \cap G^{\prime} E\right)=1 .
$$

Hence, by ([5], p. 63), there exists a complement $S$ of $R_{2}$ in $F$ which contains $F \cap G^{\prime} E$. Thus

$$
F=R_{2} \otimes S
$$

By $(g)$ therefore

$$
G=F E=R_{2} S E=R_{2}\left(S G^{\prime} E\right)
$$

and by Dedekind's Modular Law

$$
R_{2} \cap S G^{\prime} E=R_{2} \cap F \cap S G^{\prime} E=R_{2} \cap S\left(F \cap G^{\prime} E\right)=R_{2} \cap S=1 .
$$

Since $R_{2}$ is part of the center of $G$, it follows that

$$
G=R_{2} \otimes S G^{\prime} E
$$

and application of $(\mathrm{h})$ shows that $R_{2}$ is artinian. Since $R_{1}$ is likewise artinian, $R$ is artinian, and we have shown

( j) Radicable subgroups of $z G$ are artinian.

Let $p$ be a prime with $\left({ }_{3} G\right)_{p} \neq 1$. Then there exists a subgroup $Z$ of order $p$ in $z G$. Consider 


$$
\begin{aligned}
\operatorname{Hom}\left(G / G^{\prime} E Z F_{p}^{p}, Z\right) & =\operatorname{Hom}\left(F G^{\prime} E / G^{\prime} E Z F_{p}^{p}, Z\right) \\
& \cong \operatorname{Hom}\left(F_{p} G^{\prime} E / G^{\prime} E Z F_{p}^{p}, Z\right) \\
& \cong \operatorname{Hom}\left(F_{p} /\left(G^{\prime} E Z F_{p}^{p} \cap F_{p}\right), Z\right)=H .
\end{aligned}
$$

By $([5]$, p. $206 ; E), H$ is an elementary abelian $p$-group and by Proposition 2.1 it is isomorphic to a group of automorphisms of $G$. Hence, by (5.c), $H$ is countable and therefore-cf. ([5], p. 208; Lemma 54.2) - the elementary abelian $p$-group

$$
F_{p} /\left(G^{\prime} E Z F_{p}^{p} \cap F_{p}\right) \text { is finite . }
$$

This implies the finiteness of $F_{p} / F_{p}^{p}$, since otherwise $\left(G^{\prime} E Z F_{p}^{p} \cap F_{p}\right) / F_{p}^{p}$ would be infinite, but, by Dedekind's Modular Law, this is equal to

$$
F_{p}^{p}\left(G^{\prime} E Z \cap F_{p}\right) / F_{p}^{p} \cong\left(G^{\prime} E Z \cap F_{p}\right) /\left(G^{\prime} E Z \cap F_{p}^{p}\right),
$$

which is an artinian elementary abelian $p$-group and hence finite, cf. ([5], p. 65; Th. 19.2). Thus

(k) $F_{p} / F_{p}^{p}$ is finite for every $p$ with $(z G)_{p} \neq 1$.

Let $S$ be the set of all elements $x$ in $F_{p}$ with $x^{p}$ in $\left({ }_{z} G\right)_{p}$. Then $S /\left({ }_{z} G\right)_{p}$ is the socle of $F_{p} /\left({ }_{z} G\right)_{p}$ and

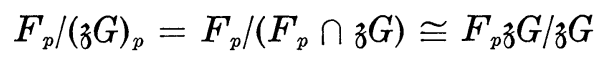

is artinian as a subgroup of the-by (5.b)-artinian group $F / z G$. Hence its socle

$$
S /\left({ }_{3} G\right)_{p} \text { is finite, }
$$

cf. ([5], p. 65; Th. 19.2). By definition of $S$ we have

$$
S^{p}=\left({ }_{\jmath} G\right)_{p} \cap F_{p}^{p} \text {. }
$$

Now

$$
\left({ }_{\jmath} G\right)_{p} / S^{p}=\left({ }_{\jmath} G\right)_{p} /\left[(z G)_{p} \cap F_{p}^{p}\right] \cong F_{p}^{p}(\jmath G)_{p} / F_{p}^{p}
$$

is finite, because, in case $(z G)_{p} \neq 1$, it is a subgroup of $F_{p} / F_{p}^{p}$, which is finite, by $(\mathrm{k})$. Therefore, if $\left({ }_{z} G\right)_{p} /\left({ }_{z} G\right)_{p}^{p}$ were infinite, $S^{p} /\left({ }_{z} G\right)_{p}^{p}$ would be infinite. But the mapping $\sigma$, defined by the rule

$$
\sigma:\left({ }_{z} G\right)_{p} x \rightarrow\left({ }_{z} G\right)_{p}^{p} x^{p} \quad \text { for } \quad x \in S
$$

is an epimorphism of the finite group $S /\left({ }_{z} G\right)_{p}$ upon $S^{p} /\left(\jmath_{z} G\right)_{p}^{p}$, implying the finiteness of $S^{p} /\left({ }_{z} G\right)_{p}^{p}$. Hence

(1) $(z G)_{p} /(z G)_{p}^{p}$ is finite for all $p$.

Since $(z G)_{p}$ is an abelian torsion group,

$$
\left({ }_{(} G\right)_{p}=A \otimes B \text {, }
$$


where $A$ is radicable and $B$ is reduced. $A$ is artinian, by $(\mathrm{j}) . B / B^{p}$ is finite, by (l), and since $B$ is reduced, $B$ is finite, by Lemma 1.1. Hence

(m) $\left({ }_{3} G\right)_{p}$ is artinian for every $p$.

By (i), there exists only a finite number of primes which are orders of elements in $G$. Hence $z G$ is the product of finitely many primary components, each of which is artinian, by $(\mathrm{m})$. This implies

(n) $z G$ is artinian.

Combination of (5.b), (e), and (n) shows that $G$ is artinian and almost abelian.

Thus (1) is a consequence of (5) which completes the proof of the Main Theorem.

REMARK. (A) It is impossible to weaken condition (3.a) by requiring only that abelian subnormal subgroups are artinian and that $G$ be a torsion group, since both this weaker condition and (3.b) are satisfied by every direct product of any number of nonabelian, finite, simple groups.

(B) The infinite cyclic group is an example of a torsionfree abelian group whose group of automorphisms is finite. This shows that conditions (4.a) and (5.a) are indispensable for the validity of the Main Theorem.

(C) A direct product of infinitely many cyclic groups of order a prime satisfies (5.a) and (5.b) showing the indispensability of condition (5.c).

6. Corollaries of the Main Theorem. The first two corollaries of the equivalence of (1), (4), and (5) of the Main Theorem, one characterizing the artinian groups with finite central quotient group, the other characterizing the finite groups by means of their automorphism groups, which will be useful in $\S 7$, may be found in [2]. We will give a proof of Corollary 6.1, since it is a simple application of the Main Theorem. It is a consequence of the equivalence of (1) and (3) of the Main Theorem that the product of all almost abelian normal subgroups of a group $G$ is almost abelian and artinian, provided its abelian subgroups are artinian (Corollary 6.4).

COROLlaRY 6.1. The following properties of the group $G$ are equivalent:

(1) $G$ is artinian with finite $G / z G$.

(2) $G$ is a torsion group and every torsion group of automorphisms of $G$ is finite.

Cf. ([2], p. 521, theorem and proof of the equivalence of (i) and (ii)).

Proof. (1) $\rightarrow(2)$. Every artinian group is a torsion group. Since 
${ }_{3} G$ is an artinian characteristic subgroup of $G$ we may, because of Lemma 1.3, apply Lemma 2.2. Thus: If $\Gamma$ is a torsion group of automorphisms of $G$ and if $\Delta$ is the subgroup of $\Gamma$ which fixes every element in $z G$ and in $G / z G$, then $\Delta$ is abelian and has finite index in $\Gamma$. The group $\Delta$ is a torsion subgroup of the group $\Sigma$ of all those automorphisms of $G$ which induce the identity in both $z G$ and $G / z G$. Application of Proposition 2.1 yields

$$
\Sigma \cong \operatorname{Hom}\left(G / z G, \jmath^{\prime} G\right) \cong \operatorname{Hom}\left(G / G^{\prime}{ }^{\prime} G,{ }_{\jmath} G\right) .
$$

Since $G$ is artinian, application of Lemma 1.3 shows that the torsion subgroup of Hom $\left(G / G^{\prime} \jmath G, \jmath G\right)$ is finite. Hence $\Delta$ is finite and $\Gamma$, as an extension of the finite group $\Gamma / \Delta$ by the finite group $\Delta$, is finite, too.

$(2) \rightarrow(1)$. If $G$ is a torsion group and if every torsion group of automorphisms of $G$ is finite, then the group of inner automorphisms of $G$ is finite. This is equivalent to the finiteness of $G / z G$. Application of the Main Theorem (equivalence of (1) and (4)) shows that $G$ is artinian.

COROLLARY 6.2. The following properties of the group $G$ are equivalent:

(1) $G$ is finite.

(2) $G$ is a torsion group and the group of automorphisms of $G$ is finite.

For a proof see ([2], p. 529, Corollary).

COROLLARY 6.3. The following properties of the abelian group $A$ are equivalent:

(1) $A$ is finite.

(2) $A$ is a torsion group and the group of automorphisms of $A$ is countable.

Proof. Evidently, (2) is a consequence of (1). Assume conversely that (2) holds. As an abelian torsion group, $A$ is the direct product

$$
A=B \otimes C
$$

of a reduced group $B$ and a radicable group $C$, see ([5], p. 63), where $C$ is a product of groups of type $Z\left(p^{\infty}\right)$. The automorphism group of a group of type $Z\left(p^{\infty}\right)$ is isomorphic to the group of $p$-adic integers, prime to $p$, cf. ([5], p. 211; Exercise 5), which is uncountable. Therefore $C=1$. Application of the Main Theorem, equivalence of (1) and (5), shows that $A$ is artinian. This implies the finiteness of $B$, cf. 
([5], p. 65; Th. 19.2). Hence $A=B$ is finite.

DEFinition. $a \mathfrak{a} G=$ product of all almost abelian, normal subgroups of $G$. This is a well determined characteristic subgroup of $G$.

COROLLARY 6.4. If every abelian subgroup of $G$ is artinian, then

(a) aaG is artinian and almost abelian;

(b) aaG contains every almost hyperabelian subnormal subgroup of $G$;

(c) Every abelian subgroup of $G / \mathfrak{a} a G$ is artinian;

(d) 1 is the only almost abelian subnormal subgroup of $G / a a G$;

(e) $\mathfrak{a} a(G / \mathfrak{a} a G)=1$.

Proof. Every epimorphic image of $a a G$ is a product of almost

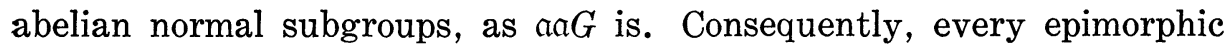
image $H$, not 1 , of $a a G$ possesses an almost abelian normal subgroup, not 1 . Therefore conditions $(3, a, b)$ of the Main Theorem are satisfied by aaG so that $\mathfrak{a} G$ is artinian and almost abelian, proving (a).

Assume that $S$ is an almost hyperabelian, subnormal subgroup of $G$. Then there exists a hyperabelian subgroup $T$ of $S$ with finite $S / T$. By hypothesis, every abelian subgroup of $T$ is artinian. Thus it follows from ([4], p. 345, Satz 6.1$)$ that $T$ is artinian, soluble and almost abelian. Consequently $T$ possesses an abelian subgroup $A$ of finite index in $T$ and $A$ has finite index also in $S$ as $S / T$ is finite. Hence $S$ is almost abelian. Thus

(f) Every almost hyperabelian, subnormal subgroup of $G$ is almost abelian.

Since $S$ is subnormal in $G$ there exists a finite chain of subgroups $S_{i}$ of $G$ such that

$$
S=S_{0}, S_{i} \text { is normal in } S_{i+1}, S_{n}=G .
$$

Clearly $S=a \mathfrak{a} S_{0}$ and $a \mathfrak{a} S_{i}$ is a characteristic subgroup of the normal subgroup $S_{i}$ of $S_{i+1}$. Since, by (a), aa $S_{i}$ is artinian and almost abelian, we have $a \mathfrak{a} S_{i} \subseteq a_{a} S_{i+1}$, so that

$$
S=\mathfrak{a} a S_{0} \subseteq \cdots \subseteq \mathfrak{a} \mathfrak{a} S_{n}=\mathfrak{a} \mathfrak{a} G,
$$

proving (b).

If $X / \mathfrak{a} a G$ is an almost abelian subgroup of $G / \mathfrak{a} a G$, then the subgroup $X$ of $G$ satisfies, by hypothesis and (a), conditions (3.a,b) of the Main Theorem so that $X$ is almost abelian and artinian. Hence $X / \mathfrak{a} a G$ is artinian, which proves (c). If $X / \mathfrak{a} a G$ is furthermore subnormal in $G / \mathfrak{a} a G$, then $X$ is an almost abelian subnormal subgroup of $G$. Hence $X \subseteq \mathfrak{a} G$, by (b), which proves (d). It also proves (e), because 
of (c) and (a), if $X$ is the well determined characteristic subgroup of $G$ with $X / \mathfrak{a} a G=\mathfrak{a} a(G / \mathfrak{a} a G)$.

7. Classes of groups. We are going to give a characterization of the class $\omega(a)$ of all artinian and almost abelian groups and of the class $\omega(f)$ of all finite groups by formal group theoretical properties.

A group which belongs to the class $\omega$ is called an $\omega$-group.

THEOREM 7.1. The class $\omega$ of groups is the class $\omega(\alpha)$ of all artinian and almost abelian groups if, and only if, $\omega$ satisfies the following properties:

I. 1 is an $\omega$-group,

II. Subgroups of $\omega$-groups are $\omega$-groups,

III. Epimorphic images of $\omega$-groups are $\omega$-groups,

IV.a An extension of an $\omega$-group by an $\omega$-group is an $\omega$-group,

V.a If $G$ is an $\omega$-group, then $G$ is a torsion group and every torsion group of automorphisms of $G$ is an w-group,

VI.a A torsion group is an $\omega$-group if all its torsion groups of automorphisms are $\omega$-groups,

VII. Every $\omega$-group is countable,

VIII. Every infinite $\omega$-group possesses an abelian subnormal subgroup, not 1 .

Theorem 7.2. The class $\omega$ of groups is the class $\omega(f)$ of all finite groups if, and only if, $\omega$ satisfies the following conditions:

I. 1 is an $\omega$-group,

II. Subgroups of $\omega$-groups are $\omega$-groups,

III. Epimorphic images of $\omega$-groups are $\omega$-groups,

IV.f Direct products of finitely many $\omega$-groups are $\omega$-groups,

V.f Every group of automorphisms of an $\omega$-group is an $\omega$ group,

VI.f A torsion group is an $\omega$-group, if its automorphism group is an $\omega$-group,

VII. Every $\omega$-group is countable,

VIII. Every infinite $\omega$-group possesses an abelian subnormal subgroup, not 1 .

Proof of the necessity of conditions I to VIII. The class $\omega(a)$ of all artinian and almost abelian groups clearly satisfies I, II, III, VII and VIII. That $\omega(a)$ is extension inherited may be found in ([3], p. 14, Folgerung 2.6) and the validity of conditions V.a and VI.a is contained in the Main Theorem, equivalence of (1) and (4).

It is evident that the class $\omega(f)$ of all finite groups satisfies I, II, III, IV.f, V.f, VII and VIII and the validity of VI.f is contained in Corollary 6.2. 
Proof of the sufficiency of conditions I to VIII. VII).

(1) Every $\omega$-group is a torsion group (provided II, IV.f, V.f,

Proof. Assume, that $G$ is an $\omega$-group, which is not a torsion group. Then $G$ contains an infinite cyclic subgroup $Z$, which is an $\omega$-group, by II. It follows, from IV.f, that $F=Z \otimes Z$ is an $\omega$-group. If $F=\{a\} \otimes\{b\}$, then automorphisms $\sigma^{\prime}$ and $\sigma^{\prime \prime}$ of $F$ are defined by

$$
\begin{aligned}
a^{\sigma^{\prime}} & =a b^{2}, & b^{\sigma^{\prime}} & =b \\
a^{\sigma^{\prime \prime}} & =a, & b^{\sigma^{\prime \prime}} & =a^{2} b .
\end{aligned}
$$

It is a consequence of V.f that $\Sigma=\left\{\sigma^{\prime}, \sigma^{\prime \prime}\right\}$ is an $\omega$-group, so that, by II, its commutator subgroup $\Sigma^{\prime}$ is an $\omega$-group, too. By its definition, $\Sigma$ is a free group of rank 2 and it is well known that $\Sigma^{\prime}$ is a free group of infinite rank. If $\Lambda$ is a free set of generators of $\Sigma^{\prime}$ and if $\Delta$ is a subset of $\Lambda$ which is neither vacuous nor $A$, then there exists one and only one automorphism of order 2 of $\Sigma^{\prime}$, which leaves every element in $\Delta$ fixed and maps every element $A-\Delta$ upon its inverse. The group of automorphisms generated by these involutions is an $\omega$-group by V.f and it is uncountably infinite by construction. This contradicts VIII and proves (1).

(2) Every finite group is an $\omega$-group (I, IV, V, VI).

Proof. 1 is an $\omega$-group, by I. Since 1 is the only group of automorphisms of the cyclic group $Z(2)$ of order 2 it follows from either of the conditions VI, that $Z(2)$ is an $\omega$-group. By either of the conditions IV, every finite, elementary abelian 2-group is an $\omega$-group. Every finite group is isomorphic to a group of automorphisms of a suitably selected finite elementary abelian 2-group. Because of $V$ therefore every finite group is an $\omega$-group.

(3.a) Every abelian $\omega$-group is artinian (II, V.a, VII).

(3.f) Every abelian $\omega$-group is finite (II, IV.f, V.f, VII).

Proof. (3.a) If $A$ is an abelian $\omega$-group, then it is, by V.a, a torsion group whose torsion groups of automorphisms are $\omega$-groups, and hence, by VII, countable. Application of the Main Theorem, equivalence of (1) and (5), shows that $A$ is artinian.

(3.f) If $A$ is an abelian $\omega$-group, then it is, by (1), a torsion group whose automorphism group is an $\omega$-group, by V.f, and hence countable, by VII. Application of Corollary 6.3 shows that $A$ is finite. (4.a) Every artinian, almost abelian group is an $\omega$-group (I, IV.a, V.a, VI.a).

Proof. By (2) every finite group is an $\omega$-group. If $A$ is artinian 
and abelian, then every torsion group of automorphisms of $A$ is finite (Lemma 1.3) and hence an $\omega$-group. VI.a shows that $A$ is an $\omega$-group. Every artinian and almost abelian group is an extension of an artinian and abelian group by a finite group. Thus, by (2), it is an extension of an $\omega$-group by an $\omega$-group and therefore, by IV.a, an $\omega$-group.

(5.a) If $\omega$ satisfies the conditions II, III, V.a, VII, VIII, then every $\omega$-group is artinian and almost abelian.

Proof. If $G$ is an $\omega$-group, then every abelian subgroup $A$ of $G$ is an $\omega$-group by II, and $A$ is artinian, by (3.a). Therefore-if we denote by $a \mathfrak{a} G$ the product of all almost abelian, normal subgroups of $G$-it follows from Corollary 6.4 that

(i) $\mathfrak{a} G$ is artinian and almost abelian, and

(ii) 1 is the only almost abelian, subnormal subgroup of $G / \mathfrak{a} G$.

If $G$ were not artinian and almost abelian, then, by (i), $G \neq a a G$ and, by (ii), $G / \mathfrak{a} a G$ is infinite. Because of III, $G / \mathfrak{a} a G$ is an $\omega$-group and VIII shows the existence of an abelian subnormal subgroup, not 1 , contradicting (ii). This contradiction shows that $G$ is artinian and almost abelian.

(5.f) If $\omega$ satisfies the conditions II, III, IV.f, V.f, VII, VIII, then every $\omega$-group is finite.

Proof. Let $G$ be an $\omega$-group and denote by If $G$ the product of all locally finite, normal subgroups of $G$. According to ([7], p. 135; Satz 28 and p. 141; Satz 40*) we have

(i) If $G$ is locally finite, and

(ii) 1 is the only locally finite subnormal subgroup of $G / \mathfrak{l} f G$. Because of III, $G / \mathrm{If} G$ is an $\omega$-group and it follows from (1) that $G$ and $G / \mathrm{l} f G$ are torsion groups.

Application of VIII and (ii) shows $G / \operatorname{lf} G=1$, so that $G=\mathfrak{l} \mathfrak{F} G$ is locally finite by (i). Every abelian subgroup of $G$ is an $\omega$-group by II, hence, by (3.a) every abelian subgroup of $G$ is finite. Application of the theorem of Kargapolov-P. Hall-Kulatilaka (see [6], p. 453; 15.4.3) yields the finiteness of $G$.

Combine (4.a) and (5.a) to get the sufficiency of the conditions in Theorem 7.1; and combination of (3.f) and (5.f) shows the sufficiency of the conditions of Theorem 7.2.

This completes the proof of the two theorems.

I am deeply indebted to Professor Reinhold Baer for his help and advice when writing this paper. 


\section{BIBLIOGRAPHY}

1. R. Baer, Groups with descending chain condition for normal subgroups, Duke Math. J. 16 (1949), 1-22.

2. Finite extensions of abelian groups with minimum condition, Trans. Amer. Math. Soc. 79 (1955), 521-540.

3. - Gruppen mit Minimalbedingung, Math. Ann. 150 (1963), 1-44.

4. — Auflösbare, artinsche, noethersche Gruppen, Math. Ann. 168 (1967), 325363.

5. L. Fuchs, Abelian groups, 3rd ed., Pergamon Press, 1960.

6. W. R. Scott, Group theory, Prentice-Hall, Inc., Englewood Cliffs, New Jersey, 1964.

7. W. Specht, Gruppentheorie, Grundlehren der mathem. Wissenschaften, Band 82, Springer Verlag, Berlin, 1956.

8. H. Zassenhaus, The theory of groups, 2nd ed., Chelsea Publishing Company, New York.

Received June 12, 1968.

Washington State University 



\section{PACIFIC JOURNAL OF MATHEMATICS}

\section{EDITORS}

H. ROYDEN
Stanford University
Stanford, California

\section{R. R. PHELPS}

University of Washington

Seattle, Washington 98105
J. DUGUNDJI

Department of Mathematics

University of Southern California

Los Angeles, California 90007

\section{RICHARD ARENS}

University of California

Los Angeles, California 90024

\section{ASSOCIATE EDITORS}

E. F. BeCKenbaCh
B. H. NEUMANN

F. WOLF
K. YoshidA

\section{SUPPORTING INSTITUTIONS}

\author{
UNIVERSITY OF BRITISH COLUMBIA \\ CALIFORNIA INSTITUTE OF TECHNOLOGY \\ UNIVERSITY OF CALIFORNIA \\ MONTANA STATE UNIVERSITY \\ UNIVERSITY OF NEVADA \\ NEW MEXICO STATE UNIVERSITY \\ OREGON STATE UNIVERSITY \\ UNIVERSITY OF OREGON \\ OSAKA UNIVERSITY \\ UNIVERSITY OF SOUTHERN CALIFORNIA
}

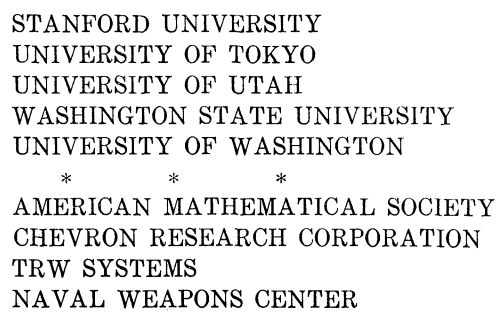

The Supporting Institutions listed above contribute to the cost of publication of this Journal, but they are not owners or publishers and have no responsibility for its content or policies.

Mathematical papers intended for publication in the Pacific Journal of Mathematics should be in typed form or offset-reproduced, double spaced with large margins. Underline Greek letters in red, German in green, and script in blue. The first paragraph or two must be capable of being used separately as a synopsis of the entire paper. It should not contain references to the bibliography. Manuscripts, in duplicate if possible, may be sent to any one of the four editors. Please classify according to the scheme of Math. Rev. 36, 1539-1546. All other communications to the editors should be addressed to the managing editor, Richard Arens, University of California, Los Angeles, California, 90024.

50 reprints are provided free for each article; additional copies may be obtained at cost in multiples of 50 .

The Pacific Journal of Mathematics is published monthly. Effective with Volume 16 the price per volume (3 numbers) is $\$ 8.00$; single issues, $\$ 3.00$. Special price for current issues to individual faculty members of supporting institutions and to individual members of the American Mathematical Society: $\$ 4.00$ per volume; single issues $\$ 1.50$. Back numbers are available.

Subscriptions, orders for back numbers, and changes of address should be sent to Pacific Journal of Mathematics, 103 Highland Boulevard, Berkeley, California, 94708.

PUBLISHED BY PACIFIC JOURNAL OF MATHEMATICS, A NON-PROFIT CORPORATION

Printed at Kokusai Bunken Insatsusha (International Academic Printing Co., Ltd.), 7-17, Fujimi 2-chome, Chiyoda-ku, Tokyo, Japan. 


\section{Pacific Journal of Mathematics \\ Vol. 29, No. $2 \quad$ June, 1969}

Bruce Langworthy Chalmers, On boundary behavior of the Bergman kernel function and related domain functionals ................... 243

William Eugene Coppage, Peirce decomposition in simple Lie-admissible power-associative rings .............................. 251

Edwin Duda, Compactness of mappings...................... 259

Earl F. Ecklund Jr., On prime divisors of the binomial coefficient......... 267

Don E. Edmondson, A modular topological lattice ............... 271

Phillip Alan Griffith, A note on a theorem of Hill ................... 279

Marcel Herzog, On finite groups with independent cyclic Sylow

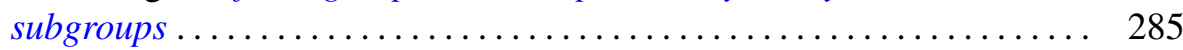

James A. Huckaba, Extensions of pseudo-valuations................. 295

S. A. Huq, Semivarieties and subfunctors of the identity functor ........ 303

I. Martin (Irving) Isaacs and Donald Steven Passman, Finite groups with small character degrees and large prime divisors. II ............ 311

Carl Kallina, A Green's function approach to perturbations of periodic

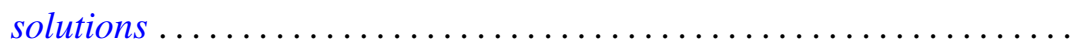

$\mathrm{Al}$ (Allen Frederick) Kelley, Jr., Analytic two-dimensional subcenter manifolds for systems with an integral ....................

Alistair H. Lachlan, Initial segments of one-one degrees ............ 351

Marion-Josephine Lim, Rank k Grassmann products ............. 367

Raymond J. McGivney and William Henry Ruckle, Multiplier algebras of

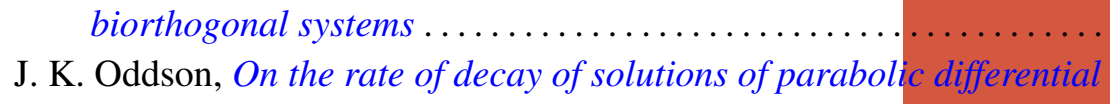

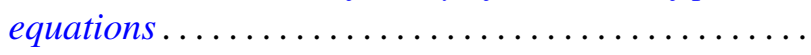

Helmut R. Salzmann, Geometries on surfaces ........... .

Annemarie Schlette, Artinian, almost abelian groups and their groups of automorphisms ............................

Edgar Lee Stout, Additional results on modules over polydisc algebras ...

Lajos Tamássy, A characteristic property of the sphere . .

Mark Lawrence Teply, Some aspects of Goldie's torsion theory. ...

Freddie Eugene Tidmore, Extremal structure of star-shaped sets ...

461

Leon Jarome Weill, Unconditional and shrinking bases in locally convex spaces... 